\title{
CARACTERIZACIÓN DE LA CULTURA INNOVATIVA EN LA COOPERACIÓN UNIVERSIDAD-EMPRESA
}

\author{
Jonathan Cohen Granados ${ }^{1 *}$, José Linares Morales² y Leonar Briceño Ariza ${ }^{3}$ \\ ${ }^{1}$ Universidad del Magdalena. Santa Marta, Colombia \\ ${ }^{2}$ Universidad Politécnica Territorial de Maracaibo. Maracaibo, Venezuela \\ ${ }^{3}$ Servicio Nacional de Aprendizaje. Santa Marta, Colombia
}

*Autor de correspondencia: jonathancohengranados@gmail.com

Recibido Enero 2020; Aceptado Marzo 2020

\begin{abstract}
Resumen - El objetivo del presente artículo es caracterizar la cultura innovativa en los procesos de cooperación universidadempresa del Departamento del Magdalena, Colombia. La investigación es de tipo descriptiva, con un diseño no experimental, transeccional y de campo; la población y muestra está constituida por 13 sujetos responsables de los procesos de cooperación universidad-empresa y cultura innovativa de la Universidad del Magdalena. Se empleó la encuesta como técnica y el cuestionario como instrumento de recolección de datos, constituido por 21 ítems bajo una escala tipo Likert, con cinco alternativas de respuesta. Fue validado por 5 expertos y calculada su confiabilidad en 0,87 según coeficiente alfa de Cronbach. En los resultados las medias aritméticas muestran una muy alta presencia de los indicadores correspondientes a los factores asociados a la cultura innovativa en la universidad. Se concluye que en la Universidad del Magdalena están dadas las condiciones para apalancar desde la cultura innovativa estrategias que permitan implementar ecosistemas de innovación que propendan la cooperación universidad-empresa y la articulación entre los diferentes actores sociales.
\end{abstract}

Palabras clave: Cultura innovativa; cooperación, universidad, empresa; ecosistema de innovación, departamento del Magdalena.

\section{CARACTERIZATION OF INNOVATIVE CULTURE IN UNIVERSITY-ENTERPRISE COOPERATION}

\begin{abstract}
The objective of this paper is to characterize the innovative culture in the university-enterprise cooperation processes of the Department of Magdalena, Colombia. The research is of a descriptive type, with a nonexperimental, transectional and field design; the population and sample is made up of 13 subjects responsible for the university-enterprise cooperation processes and innovative culture of the Universidad del Magdalena. The survey was used as a technique and the questionnaire as a data collection tool, consisting of 21 items under a Likert scale, with five response alternatives. It was validated by 5 experts and calculated its reliability at 0.87 according to Cronbach's alpha coefficient. In the results, arithmetic means show a very high presence of indicators corresponding to the factors associated with the innovative culture in the university. It is concluded that at the Universidad del Magdalena the conditions are in place to leverage from innovative culture strategies to implement innovation ecosystems that promote university-enterprise cooperation and articulation between different social actors
\end{abstract}

Keywords: Innovative culture, cooperation, university, enterprise, innovation ecosystem, department of Magdalena. 


\section{Introducción}

En tiempos de globalización, las empresas atraviesan por una serie de transformaciones impulsadas por la dinámica del mercado y el desarrollo tecnológico, para lo cual requieren de talento humano capacitado para enfrentarlos; lo cual representa nuevos retos para las universidades en cuanto a su función dentro de la sociedad, principalmente en lo relacionado con la vinculación universidad-empresa a objeto de promover la innovación empresarial como base de su competitividad.

Al respecto, Ankrah \& Omar (2015) refieren que la cooperación entre Universidad y Empresa no es un fenómeno reciente y se refiere a la interacción que apunta de manera fundamental a promover el intercambio de conocimiento y tecnología entre los mismos. En ese mismo sentido Hammert et al. (2014) plantea que la colaboración universidad-industria es un modo de innovación cada vez más importante, dado que permite que tanto universidades como empresas puedan aprovechar habilidades complementarias y disminuir costos de I+D al compartir recursos.

También, el Manual de Oslo (OCDE, 2005) establece los tipos de innovación empresarial, y en este contexto, la vinculación con la universidad emerge como alternativa para el logro de los objetivos organizacionales planteados, estimulando la oportunidad de innovación de los procesos de negocio, la estructura organizacional y los modelos de gestión. En consecuencia, la cooperación universidad-empresa mejora el desempeño innovador con base en la transferencia del conocimiento y la tecnología generada en la universidad, lo cual definitivamente contribuye al proceso de innovación empresarial (Palacio et al., 2017; Paternina-Pedroza et al., 2019; Vélez et al, 2019; Gutiérrez et al., 2019).

En ese sentido, las empresas que usan a la Universidad como fuente de información obtienen un alto desempeño innovador; no obstante, en estas organizaciones económicas se motorizan transformaciones que a su vez conllevan cambios institucionales de las universidades, y en materia de investigación, las autoridades académicas comienzan a reconocer que el conocimiento se comercializa, por lo que promueven mecanismos de licenciamiento, patentes, transferencia y cesión de derechos de autor (FongSilva et al., 2019; García Galván, 2018; Geizzelez \& Linares, 2016).

Desde esta perspectiva, la cultura innovativa destaca como un importante elemento y de gran impacto para la cooperación universidad-empresa, definiéndose esta como la conducta desarrollada a nivel organizacional, apalancada por la creatividad de sus integrantes, dentro de un ambiente de trabajo estimulante de la producción de ideas novedosas con una alta motivación al logro, para satisfacer las necesidades de conocimiento dentro de una dinámica altamente competitiva (Ramos \& Moog, 2014); como resultado de enfrentar la incertidumbre y el riesgo al romper paradigmas, por lo que son las personas el eje central de esta cultura (Souto, 2015; Aldana, 2017).

De esta manera, la cultura de innovación abarca el conjunto de conocimientos, prácticas y valores que determinan posturas individuales y colectivas que promueven la generación de nuevos conocimientos, con base en la creatividad, el trabajo en equipo, la cooperación, la autonomía, la tolerancia al riesgo, el aprendizaje continuo, la iniciativa emprendedora, la suficiencia de recursos, la orientación estratégica hacia el cliente, el compromiso, la toma de decisiones, la participación de los trabajadores y la flexibilidad (Cornejo \& Muñoz, 2009; Labarca, García \& Villegas, 2012; Camio, 2014; Naranjo \& Calderón, 2016; Araya-Pizarro, 2019).

IPSA SCIENTIA - Vol. 5 No $1-2020$ 
A ello, se suman los efectos de la globalización en las actividades sociales y económicas que ponen a las universidades, ante la necesidad de lograr estándares adecuados a los que se tienen a nivel global. Todo esto, conduce a que la cooperación entre las universidades para enfrentar estas oportunidades y desafíos sea cada vez más relevante.

En Colombia históricamente se asoció la relación Universidad-Empresa con términos como alianza, cooperación, transferencia de conocimiento; y durante mucho tiempo, la relación más sencilla que surgió entre las universidad y sector productivo se basó en la vinculación de estudiantes en pasantías y/o prácticas profesionales, un tipo de cooperación que permitió en muchos casos ofrecerle oportunidades laborales a los recién graduados. Otro modo de cooperación fue la concreción de convenios interinstitucionales para la formación, educación y capacitación de recurso humano; y también la que se desprende de alianzas de cooperación o contratos de consultoría ejecutados por la empresa.

Este status en la relación Universidad-Empresa ha empezado a ser tratado con más atención por los hacedores de políticas públicas, y cuenta de ello dan los espacios que han sido creados en alianzas que incluyen al sector público (Linares-Morales \& Geizzelez-Luzardo, 2016). También se han dado avances potenciados por la Política Nacional de Ciencia, Tecnología e Innovación que entre otras bondades busca replicar la estrategia de Parques Científicos y Tecnológicos implementada en países como Brasil y Estados Unidos.

En ese contexto, desde finales del Siglo XX particularmente en el departamento del Magdalena ha existido más una articulación que una cooperación entre la Universidad y el sector productivo, la cual respondía a una dinámica natural propia de la oferta y demanda de mano de obra cualificada y no como una estrategia que buscara el desarrollo de proyectos de ciencia, tecnología e innovación. Es así que han surgido desde la Universidad del Magdalena propuestas como la de generar espacios de cooperación con el sector productivo, participar en el diseño de la política departamental y distrital de innovación, y crear unidades organizativas e infraestructura para la innovación y el emprendimiento. Tales iniciativas han tenido un impacto en la generación de procesos de innovación y cooperación con el sector privado.

Para que se implemente la cultura de innovación en la universidad, es importante contar con ecosistemas de innovación y cooperación basados en redes, involucrando diferentes actores del sector empresarial, la sociedad y el Estado. La innovación como cultura organizacional sustentada en procesos humanos constituye una estrategia de cooperación Universidad-Empresa para fomentar valores institucionales en armonía con el entorno (Flores Urbáez, 2015). De allí, surge la necesidad e interés del presente artículo, a fin de realizar una caracterización de la cultura innovativa como estrategia de cooperación universidad-empresa en las instituciones de educación superior del Departamento del Magdalena, Colombia.

\section{Materiales y Métodos}

Por su naturaleza, se considera una investigación descriptiva, bajo un diseño no experimental transeccional de campo (Hernández et al., 2014; Tamayo y Tamayo, 2012). A continuación se presenta en la tabla 1 la caracterización de la población objeto de investigación. Es importante acotar que, la misma corresponde a la Universidad del Magdalena, por ser la institución pública (única del sector) insignia del departamento a nivel universitario, que cuenta con convenios suscritos con entes públicos y privados, así como una amplia trayectoria en programas de investigación y extensión fortalecidos por la existencia de

IPSA SCIENTIA - Vol. 5 No $1-2020$ 
instancias organizacionales responsables de su gestión, y está conformada por 13 sujetos responsables de los procesos de cooperación universidad-empresa y cultura innovativa.

Tabla 1: Caracterización de la población

\begin{tabular}{|c|c|c|c|}
\hline Procesos clave & Unidad Organizacional & Departamento & $\begin{array}{c}\text { Cantidad de } \\
\text { sujetos }\end{array}$ \\
\hline \multirow{3}{*}{$\begin{array}{l}\text { Cultura } \\
\text { Innovativa }\end{array}$} & \multirow{3}{*}{$\begin{array}{l}\text { Vicerrectoría de } \\
\text { Investigación }\end{array}$} & Centro de innovación y emprendimiento & \multirow{3}{*}{5 sujetos } \\
\hline & & $\begin{array}{c}\text { Dirección de transferencia de conocimiento y } \\
\text { propiedad intelectual }\end{array}$ & \\
\hline & & $\begin{array}{c}\text { Dirección de gestión del } \\
\text { conocimiento }\end{array}$ & \\
\hline \multirow{4}{*}{$\begin{array}{l}\text { Cooperación } \\
\text { universidad } \\
\text { empresa }\end{array}$} & \multirow{4}{*}{$\begin{array}{c}\text { Vicerrectoría de } \\
\text { Extensión y Proyección } \\
\text { Social }\end{array}$} & Dirección de desarrollo social y productivo & \multirow{4}{*}{8 sujetos } \\
\hline & & $\begin{array}{c}\text { Dirección de proyección } \\
\text { Cultural }\end{array}$ & \\
\hline & & $\begin{array}{c}\text { Dirección de prácticas } \\
\text { profesionales }\end{array}$ & \\
\hline & & $\begin{array}{l}\text { Centro de } \\
\text { egresados }\end{array}$ & \\
\hline & & $\begin{array}{l}\text { Total de sujetos } \\
\text { de la población }\end{array}$ & 13 sujetos \\
\hline
\end{tabular}

Fuente: Elaboración propia (adaptado de sitio oficial Unimagdalena, 2019)

Se tomó como muestra el 100\% de la población general, teniendo en cuenta que la población es pequeña y accesible, en este sentido la muestra objeto de estudio se aborda a través de un censo poblacional, La técnica de recolección de datos fue la observación mediante encuesta, siendo el instrumento un cuestionario, de 21 ítems, tipo escala Likert, con cinco alternativas de respuesta: Totalmente de acuerdo (TA); de acuerdo (DA); ni de acuerdo ni en desacuerdo (NAND); en desacuerdo (ED); y totalmente en desacuerdo (TED). Se sometió a validez de contenido, mediante el juicio de 5 expertos; su confiabilidad se calculó mediante el coeficiente alfa de Cronbach una vez realizada la prueba piloto respectiva, a un grupo de 6 sujetos con características similares a la muestra, que laboran en otras universidades del Magdalena, obteniendo como resultado 0.87, valor considerado de alta/muy alta confiabilidad (Hernández et al. 2014).

Se aplicó la estadística descriptiva en el análisis de los datos, mediante la representación de frecuencias absolutas y relativas; con estos datos se procedió al cálculo de la media aritmética para cada uno de los indicadores de la dimensión considerada, con apoyo del software Microsoft Excel 2010. Se realizó la tabulación respectiva y se interpretaron los datos porcentuales para determinar el nivel de presencia del indicador dentro de la muestra estudiada, cuyas categorías de valoración se muestran en la tabla 2.

Tabla 2: Baremo de interpretación de la media aritmética por indicador

\begin{tabular}{|c|c|c|}
\hline \multicolumn{2}{|c|}{ Rangos de la media aritmética } & \multirow{2}{*}{ Categoría } \\
\hline Límite superior & Límite inferior & \\
\hline 5,00 & 4,21 & Muy Alta Presencia (MAP) \\
\hline 4,20 & 3.31 & Alta Presencia (AP) \\
\hline 3.30 & 2.41 & Moderada Presencia (MP) \\
\hline 2.40 & 1.51 & Baja Presencia (BP) \\
\hline 1.50 & 1.00 & Muy Baja Presencia (MBP) \\
\hline
\end{tabular}

Fuente: Elaboración propia 


\section{Resultados y Discusión}

Se presenta a continuación el análisis y discusión de los resultados obtenidos tras la interpretación de los datos arrojados por el cuestionario; es así como, en la tabla 3 se ilustran los datos relacionados con los Factores asociados a la cultura innovativa, siendo estos: (1) ambiente creativo; (2) trabajo en equipo; (3) confianza en el personal; (4) liderazgo innovador; (5) comunicación; y (6) competitividad.

Tabla 3: Resultados del estudio respecto a los factores asociados a la cultura innovativa

\begin{tabular}{cccccccc}
\hline \multirow{2}{*}{ Indicador } & \multicolumn{9}{c}{ Alternativas de respuesta } & \multicolumn{2}{c}{ Media Aritmética } \\
\cline { 2 - 8 } & TA & DA & NAND & ED & TED & \multirow{2}{*}{ Valor } & \multirow{2}{*}{ Categoría } \\
\hline Ar(\%) & $\operatorname{Fr}(\%)$ & $\operatorname{Fr}(\%)$ & $\operatorname{Fr}(\%)$ & $\operatorname{Fr}(\%)$ & & MAP \\
Trabajonte creativo equipo & 61,54 & 28,21 & 5,13 & 0,00 & 0,00 & 4,67 & MAP \\
Confianza en el personal & 61,54 & 28,21 & 5,13 & 0,00 & 0,00 & 4,67 & MAP \\
Liderazgo innovador & 41,03 & 48,72 & 10,26 & 0,00 & 0,00 & 4,33 & MAP \\
Comunicación & 61,54 & 28,21 & 5,13 & 0,00 & 0,00 & 4,67 & MAP \\
Competitividad & 61,54 & 28,21 & 5,13 & 0,00 & 0,00 & 4,67 & MAP \\
\hline Promedio General & 71,79 & 23,08 & 5,13 & 0,00 & 0,00 & 5,00 & MAP \\
\hline
\end{tabular}

Fuente: Elaboración propia

En relación al indicador Ambiente Creativo, el 61,54 \% de los encuestados, se expresó Totalmente de acuerdo (TA) con que, como parte de la cultura innovativa, se estimula la creatividad en los trabajadores de la universidad, apoyando a quienes aportan ideas innovadoras siendo promovidos a nuevos cargos; el promedio para este indicador fue de 4,67, lo que manifiesta una Muy Alta Presencia (MAP), dado que en la universidad existe un ambiente creativo, esto es, un espacio alrededor de cada persona para sus iniciativas y hacer fluir la creatividad. Ser creativo se relaciona con la competencia para hacer asociaciones, establecer relaciones, combinar e integrar ideas y conceptos de una forma diferente o única, cuyo resultado sea disruptivo; por tanto, para ser innovador se requiere tomar una idea creativa y convertirla en un bien que sea de utilidad. (Hernández Arteaga et al., 2015; Quintero Ramos et al., 2018).

Con respecto al indicador Trabajo en equipo, el 61,54\% de los encuestados expresaron encontrarse Totalmente de acuerdo (TA) ante el hecho de que en la universidad existe la colaboración entre personas para la consecución de resultados comunes; para ello, los equipos de trabajo se integran para colaborar activamente. La media aritmética obtenida fue de 4,67, lo que indica una Muy Alta Presencia (MAP) en la universidad. Estos resultados se corresponden con los planteamientos de Souto (2015), quien resalta la importancia de la cooperación e interacción entre las personas, dentro de un entorno abierto y participativo; por lo que el éxito del equipo radica en aunar esfuerzos, conocimientos y habilidades hacia los objetivos comunes.

Respecto al indicador Confianza en el Personal, se obtuvo que el 48,72 \% de los encuestados expresó estar De acuerdo (DA) con el hecho de que en la universidad existe un clima de familiaridad entre el personal que allí labora; brindando confianza en sus trabajadores para desarrollar con autonomía sus labores; la media aritmética obtenida para este indicador fue de 4,33, lo que evidencia una Muy Alta Presencia (MAP), De esta manera, la confianza dentro de la institución es generada por las actitudes positivas y los comportamientos de ciudadanía y de apoyo desplegados por todos sus integrantes (Arancibia Carvajal et al., 2015; Omar et al., 2016). 
Para el indicador Liderazgo Innovador el 61,54 \% de los encuestados optó por la opción Totalmente de acuerdo (TA); alegando que la universidad ofrece capacitación a los empleados para asumir el liderazgo en nuevos procesos, fomentando el espíritu innovador. Este indicador alcanzó una media aritmética de 4,67, lo que indica una Muy alta presencia (MAP), lo cual evidencia que en la institución se ejerce un liderazgo innovador, caracterizado por elementos tales como el establecimiento de retos, y no objetivos; la definición de visiones inspiradoras; gestionar con base en la confianza; y dirigir mediante autoridad, entre otros aspectos (Caldera, 2015).

En cuanto al Indicador Comunicación, se observa que el 61,54 \% de los encuestados se expresaron Totalmente de acuerdo (TA) respecto a que en la universidad existe una excelente comunicación entre todos los empleados, apoyada en medios digitales. La media aritmética fue de 4,67, equivalente a una Muy Alta Presencia (MAP) del indicador. Estos resultados evidencian que la comunicación en las instituciones educativas, basada en la participación activa del profesorado, como elemento de cultura innovativa, constituye uno de los mejores instrumentos para una mejor gestión de los procesos de cooperación universidad-empresa (Medina et al., 2015).

Por último, para el indicador Competitividad, se encontró que el 71,79 \% de los encuestados se mostró Totalmente de acuerdo (TA), afirmando que la universidad incorpora nuevos procesos determinantes de competitividad, desarrollando y manteniendo ventajas comparativas que le permiten sostener una posición destacada en su entorno de actuación, gracias a los esfuerzos de un equipo directivo dinámico, actualizado, abierto al cambio organizativo y tecnológico. La media aritmética de este indicador fue de 5,00, representando una Muy Alta Presencia (MAP), reafirmando que la competitividad involucra la innovación, incorporando nuevos procesos, productos y servicios (Aldana, 2017).

\section{Conclusiones}

Al revisar los factores asociados a la cultura innovativa se concluye que en la universidad existe un ambiente creativo, el trabajo en equipo se considera un factor innovador, existe un clima de familiaridad en el personal que allí labora; se le otorga la confianza necesaria a l talento humano para desarrollar con autonomía sus labores, el liderazgo innovador forma parte de la cultura innovadora, se promueve una comunicación efectiva como resultado de un entendimiento mutuo, y se incorporan nuevos procesos clave para incrementar la competitividad.

Es por ello que, se sugiere a la Universidad del Magdalena, mantener su reconocimiento al valor e importancia de los factores asociados con la cultura innovativa en el marco de las acciones del proceso de cooperación Universidad-Empresa, pues estos impactan el ámbito empresarial y en la obtención de soluciones novedosas, y el rediseño de los procedimientos de trabajo. Es recomendable consolidar y promover la creatividad como un valor preponderante dentro de la cultura innovativa de cualquier organización, por cuanto a través de ello se podrá generar innovaciones que impulsen el crecimiento de la organización y despierten el interés y confianza del sector empresarial, mediante políticas de incentivo al personal para la creación y desarrollo de nuevo conocimiento científico y tecnológico; por medio del reconocimiento al logro, promociones de cargo, y bonificaciones especiales.

Finalmente, es importante aumentar la calidad del proceso de toma de decisiones de la universidad, mediante evaluaciones antes, durante y después de haberlas tomado, para supervisar los resultados obtenidos y mantener al mínimo los posibles riesgos que puedan influir negativamente en los mecanismos

IPSA SCIENTIA - Vol. 5 No $1-2020$ 


\section{Caracterización de la cultura innovativa en la cooperación universidad-empresa}

de transferencia de tecnología y conocimiento integrados en la estrategia de cooperación universidadempresa.

\section{Referencias}

Aldana, G. (2017). Cultura innovativa en la organización de la dirección de tecnología de información y comunicación de la Universidad del Zulia. REVECITEC, 7(2), 73-96.

Arancibia Carvajal, S., Donoso Pérez, M., Venegas Cabello, R., \& Cárdenas Espinosa, C. (2015). Identificación de factores clave en la cultura de innovación: El caso de la mediana minería en Chile. Journal of technology management \& innovation, 10(1), 132-145.

Ankrah, S. \& Omar, AT (2015). Colaboración entre universidades e industria: una revisión sistemática. Revista escandinava de gestión, 31(3), 387-408.

Araya-Pizarro, S. (2019). Cultura innovativa de una escuela de turismo en Chile. Estudios y perspectivas en turismo, 28(2019), $167-184$.

Caldeira, M. (2015). Liderazgo innovador para emprendimientos sociales en organizaciones sin fines de lucro. CICAG, 13(1), 191-207.

Camio, M. I. (2014). El rol de los aspectos emocionales en la gestión de la cultura innovadora. Revista de la Facultad de Ciencias Económicas: Investigación y Reflexión, 22(2), 9-28.

Cornejo, M. \& Muñoz; E. (2009). Percepción de la innovación: cultura de innovación y capacidad innovadora. Pensamiento iberoamericano, (5), 121-147.

Donawa Torres, Z. A, \& Gámez Araujo, W. G. (2019). Ausencia de habilidades gerenciales para una cultura de innovación universitaria en instituciones de Colombia y Venezuela. Universidad \& Empresa, 21(36), 8-35.

Flores Urbáez, M. (2015). La innovación como cultura organizacional sustentada en procesos humanos, Revista Venezolana de Gerencia, 20(70), 355-371.

Fong-Silva, W., Taron-Dunoyer, A. \& Zabaleta-Mesino, R. (2019). Nuevo liderazgo organizacional para fortalecer instituciones universitarias débilmente acopladas según Weick. IPSA SCIENTIA: Revista Científica Multidisciplinaria, 4(1), 60-70.

García Galván, R. (2018). El papel de las instituciones y de la colaboración universidad-empresa en el desarrollo: evidencias de la UAEMÉX y la UABC. Paradigma EconóMico, 10(1), 81-118.

Geizzelez, M. \& Linares, J. (2016). Transferencia tecnológica para la vinculación con las comunidades en el Programa Nacional de Formación en Informática. REVECITEC, 6(2), 1-14.

Gutiérrez, P., Benavente, L. \& Cruz, L. (2019). La cooperación universidad-empres y su influencia sobre el desempeño innovador empresarial de la industria manufacturera de Perú. RISTI, (E23), 469-480.

Hammert, M.; Bstieler, L. \& Okamuro, H. (2014). Reducir la brecha cultural: formación de confianza en colaboraciones de investigación universidad-industria en los EE. UU., Japón y Corea del Sur. Technovation, 34(10), 605-616.

Hernández Arteaga, I.; Alvarado Pérez, J. C. \& Luna, S. M. (2015). Creatividad e innovación: competencias genéricas o transversales en la formación profesional. Revista Virtual Universidad Católica del Norte, 44, 135-151.

Hernández, R., Fernández, C., \& Baptista, P. (2014). Metodología de la Investigación. México D.F:: McGraw-Hill

IPSA SCIENTIA - Vol. 5 No $1-2020$ 


\section{Caracterización de la cultura innovativa en la cooperación universidad-empresa}

Iglesias, M. \& Rodicio, M. (2013). El desarrollo de la creatividad e innovación. Un reto ante la crisis actual. Revista de investigación en educación, 11(1), 134-148.

Labarca, N., García, J. \& Villegas, E. (2012). Cultura de innovación como herramienta de competitividad en la gestión directiva universitaria venezolana. Ominia, 18(2), 83-94.

Linares-Morales, J. \& Geizzelez-Luzardo, M. (2016). Políticas científicas y tecnológicas para la gestión del conocimiento en instituciones universitarias. IPSA SCIENTIA: Revista Científica Multidisciplinaria, 1(1), 10-17.

Medina, F., Hernández, E. \& Monsalve, L. (2015). Medios de comunicación utilizados en los centros educativos para difundir los procesos de innovación docente. Revista Lasallista de Investigación, 12(2), 45-53.

Naranjo, j. \& Calderón, G. (2016). Construyendo una cultura de innovación: una propuesta de transformación cultural. Estudios gerenciales, 31(135): 223-236.

OCDE (2005). Manual de Oslo; Guía para la Recogida e Interpretación de Datos Sobre Innovación (3ra. ed.). Madrid: Grupo Tragsa.

Omar, A., Salessi, S. \& Urteaga, F. (2016). Liderazgo, confianza y flexibilidad laboral como predictores de identificación organizacional: Un estudio con trabajadores argentinos. Pensamiento Psicológico, 14,(2), 33-47.

Palacio, A., Arévalo, P. \& Guadalupe; J. (2017). Tipología de la Innovación Empresarial según Manual de Oslo. CienciAmérica: Revista de divulgación científica de la Universidad Tecnológica Indoamérica, 6(1), 97-102.

Paternina-Pedroza, H., Linares-Morales, J. \& Hernández-Ayala, K. (2019). Transferencia de tecnología y conocimiento en el sector exportador de bovinos. IPSA SCIENTIA: Revista Científica Multidisciplinaria, 4(1), 10-20.

Quintero Ramos, M., Morillo Olarte, E. \& González Martínez, J. (2018). Elementos de la cultura innovativa en el comercio de prendas de vestir del municipio Maicao - Colombia. Neumann Business Review, 4(1), 4-27. https://doi.org/10.22451/3002.nbr2018.vol4.1.10017

Ramos, C. \& Moog, M. (2014). Cultura innovativa para una investigación universitaria socialmente responsable. Revista Professare, 3(1), 42-59.

Souto, J. E. (2015). Gestión de una cultura de innovación basada en las personas. Journal of technology management \& innovation, 10(3), 60-65.

Tamayo y Tamayo, M. (2012) El proceso de investigación científica. Incluye evaluación y administración de proyectos de investigación. Limusa. Noriega Editores.

Velez, C.I., Afcha, S.M., \& Bustamante, M.A.. (2019). Cooperación universidad - empresa y su efecto sobre el desempeño innovador empresarial. Información tecnológica, 30(1), 159-168. https://dx.doi.org/10.4067/S0718-07642019000100159 